\title{
Expansión de la mancha urbana de la Ciudad de Chihuahua 2000-2012
}

Expansion of the urban area of Chihuahua's city 2000-2012

\author{
Orozco González Clara Patricia ${ }^{1 凶}$, Porras Flores Damián A. ${ }^{2}$. Ortega Rodríguez Anabel ${ }^{2}$. \\ González Aldana Ricardo A. García González Cesar G. ${ }^{2}$. \\ ${ }^{1}$ Estudiante de posgrado en Planificación y Desarrollo Urbano de la UACJ. ${ }^{2}$ Profesor de la \\ Facultad de Ciencias Agrotecnológicas, Universidad Autónoma de Chihuahua Campus I, \\ Chihuahua, Chih., México. Tel. (614)4391844. \\ $\bowtie$ Autor para correspondencia: claraog.20@gmail.com
}

Recibido: $18 / 02 / 2017$

Aceptado: 30/06/2017

\section{RESUMEN}

En el presente trabajo se pretende dar a conocer el acelerado crecimiento de la mancha urbana de la ciudad de Chihuahua que se ha generado en tan solo diez años, mediante los Sistemas de Información Geográfica lo que hace una investigación novedosa e interesante, con estos sistemas podemos ver que, si bien la ciudad ha tenido un crecimiento en su espacio, económico y social no se ha dado un desarrollo equilibrado sobre su territorio. Por lo que el objetivo de esta investigación es conocer las acciones políticas, sociales, ambientales y económicas, que produjeron la expansión de la mancha urbana en sus diferentes puntos cardinales de la Ciudad en los últimos 10 años, representados con los SIG. Generado problemáticas sobre todo en el tema de los asentamientos humanos en espacios carentes de características viables para una buena calidad de vida, dejando consecuencias en la estructura de la ciudad, tanto física como social, puesto que no se dio una adecuada planeación para el crecimiento de la mancha urbana.

Palabras clave: Expansión Urbana. Planeación Urbana. Sistemas de Información Geográfica.

\begin{abstract}
In the present work we intend to present the accelerated growth of the urban spot of Chihuahua's city, that has been generated in only ten years, through the Geographic Information Systems which makes a novel and interesting research, with these systems we can See that although the city has had a growth in its space, economic and social has not given a balanced development on its territory. So the objective of this research is to know the political, social, environmental and economic actions that led to the expansion of the urban spot in its different cardinal points of the City in the last 10 years, represented by GIS. Generated mainly problematic in the subject of
\end{abstract}


human settlements in spaces lacking viable characteristics for a good quality of life, leaving consequences in the structure of the city, both physical and social, since there was given an adequate planning for the growth of the urban area.

Keywords: Urban Expansion. Urban Planning. Geographic information systems.

\section{INTRODUCCIÓN}

La adecuada Planeación y Gestión de una ciudad nos dará como consecuencia un Ordenamiento Territorial armónico, equilibrado y sustentable.

Como se ha observado en Estados Unidos de América y Europa, siendo estos los países pioneros en el tema de Ordenación Territorial, después de las consecuencias de la Revolución Industrial como la motorización de los automóviles y todos los cambios que esto trajo a la sociedad y las ciudades, preocupándose por cumplir las nuevas necesidades (Fernández, 2006).

En Europa se crea la Carta Europea de Ordenación Territorial en 1994 siendo el pionero en este tema, se empieza a preocupar y a profundizar más en la Ordenación de sus ciudades. Sabiendo que la ordenación del territorio se compone de muchos elementos que se encuentran en su entorno y que sin la ayuda de estos no se podrá llegar al objetivo que será la planeación ordenada de su territorio. Por lo que a continuación se presentan algunos de los elementos que se encuentran dentro de un territorio, formando un sistema Territorial:

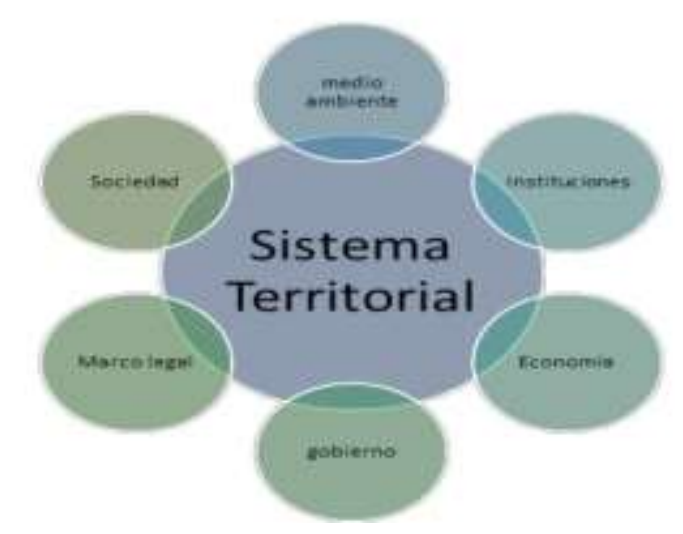

Figura 1 Elementos que forman un Sistema Territorial

Basados en estos elementos tenemos que trabajar en las necesidades de cada territorio, ya que son diferentes por su cultura, economía, sociedad, medio geográfico y ambiente (Gómez, 2007).

La planeación urbana tiene como objetivo rescatar y modernizar la ciudad antigua y ordenar y restaurar el territorio mediante estrategias tales como la creación de redes viales, rehabilitación del centro histórico y el mejoramiento de la imagen urbana, la descentralización de la vivienda y las reglamentaciones en cuanto aspectos de salubridad y el precio de los terrenos (Lacomba, 2004).

La expansión de la mancha urbana del municipio de Chihuahua ha tenido un acelerado crecimiento en los últimos diez años puesto que su población para el año 2000 era de 671,790 hab. Y para el año 2010 se contaba con 819,543 hab. (INEGI, 20022010), trayendo consigo nuevas necesidades y problemáticas, una de ellas, los 
asentamientos humanos implantados en espacios que muchas veces no cumplen con los requisitos para una calidad de vida deseable.

\section{MARCO TEÓRICO}

La Expansión Urbana se entiende como el desarrollo urbano disperso, difuso, separado de la periferia de las ciudades, descoordinado $\mathrm{y}$ sin tomar en cuenta los efectos sociales y ambientales que produce (San Miguel, 2010).

Existen dos variables que son importantes para nuestra investigación que son el Espacio y el Territorio, en los que desenvolvemos nuestras actividades diariamente.

ESPACIO.- es la proporción delimitada por una línea que puede ser imaginaria o funcional.

TERRITORIO.- constituye un espacio concreto $\mathrm{y}$ específico en términos referenciales, en la medida que al vincularse con base en relaciones políticas, al territorio estructurado, que ya presenta límites administrativos y estructura de poder, adquiere características de región delimitada (Torres, 2008).

USOS DE SUELO: El objeto de la Normativa del Uso del Suelo Urbano de la Ciudad de Chihuahua es proponer un marco general que, de manera uniforme, sirva para organizar el espacio de una diversidad de asentamientos humanos, indiferentemente de su tamaño y localización.

Desarrolla el tratamiento sistemático del conjunto compuesto por la caracterización del suelo urbano, el uso del suelo para las funciones urbanas, las condiciones generales del uso del suelo urbano y las condiciones particulares de la habilitación del suelo urbano (ver figura 2). El uso de suelo normalmente se refiere al régimen de manejo al cual somete el hombre un sitio y el concepto de Cobertura de Suelo es un descriptor del estado de la vegetación en un sitio (Peña, 2005).

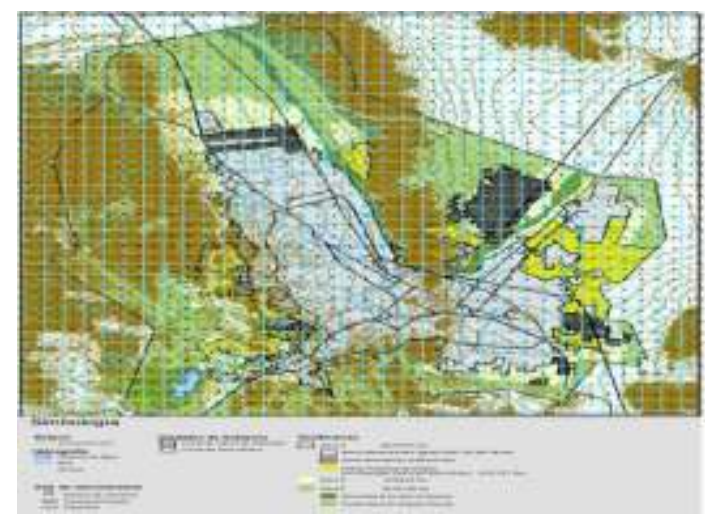

Figura 2 Zonificación Primaria, fuente IMPLAN 2007

\section{MATERIALES Y METODOS}

La investigación se llevó acabo en la Facultad de Ciencias Agrotecnológicas, durante los meses de Enero-Agosto del año 2013.

\section{MATERIALES}

En la que se utilizaron como materiales necesarios el software de Sistemas de Información Geográfica ArcGis 9.2 y Google Earth avanzado. Donde en ambos sistemas podemos almacenar, diagnosticar, analizar, gestionar y manipular la información deseada, ya que se divide en varias capas de información, en las que podemos visualizar el relieve del territorio, las imágenes

satelitales de la actualidad y cronológicamente del pasado, las divisiones de las vías carreteras, los cuerpos de agua, siendo estos las principales funciones de los sistemas. 


\section{METODOS}

La investigación fue llevada mediante estudios cuantitativos y cualitativos, debido a las propias características de descripción de la problemática estudiada, con información estadística del Municipio de Chihuahua, como densidad de población, información por AGEBS, tipo de suelo, entre otros datos, para así poder representar la información obtenida en los Sistemas de Información Geográfica.

\section{RESULTADOS}

Se realizó una cronología del crecimiento urbano de los periodos 2000, 2005 y 2012 para poder resaltar la expansión de la mancha urbana. De los nuevos Desarrollos habitacionales en toda la ciudad de Chihuahua del año 2000 al 2012, se estudiaron 4 Fraccionamientos con mayor problemática, los que dan como resultado la expansión de la mancha urbana al asentarse en la periferia de la ciudad, generando problemáticas entre la sociedad, el ambiente y los entes gubernamentales encargados de proveer los servicios básicos y el equipamiento residencial.

\section{Cronología del crecimiento de la mancha urbana}

En un análisis realizado por el IMPLAN en el año 2000 se observa que el crecimiento urbano se ha dado aceleradamente debido a su topografía plana y la facilidad para dotar de servicios (disponibilidad de agua) la cual se extrae de la presa Chihuahua. La mancha urbana para el año 2000 aún se ve consolidada pero ya con un notable énfasis hacia el norte de la ciudad (ver mapa 1).

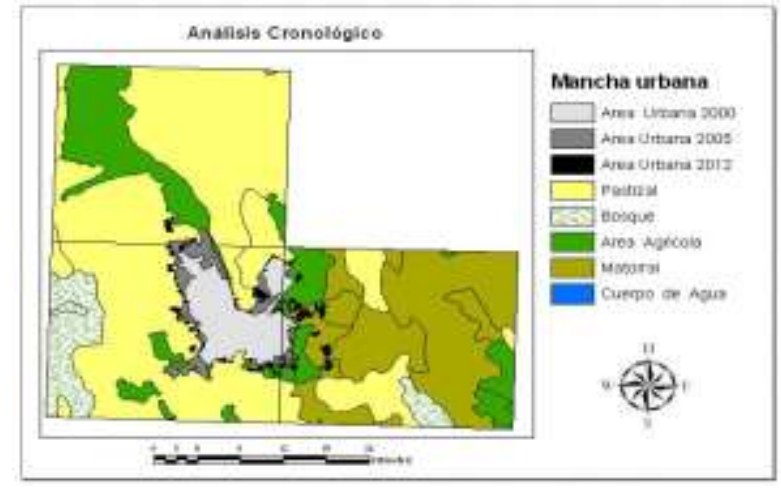

Mapa 1 Análisis Cronológico de los años 2000,2005 y 2012

\section{Situación actual de la Mancha Urbana con los Sistemas de Información Geográfica}

Su carácter de ciudad capital y su impulso en el desarrollo económico e industrial, ha generado una migración de las poblaciones rurales hacia esta urbe ubicada en el centro del Estado, este proceso migratorio ha incidido en el crecimiento de la población (ver figura 3), la diversificación de las actividades económicas, la ocupación de mayores superficies del suelo y la dinámica constante de crecimiento de la Ciudad, que se ha manifestado en una modificación sustancial del entorno original, y que como resultante ha impactado los procesos naturales del ecosistema en que se asienta la ciudad.

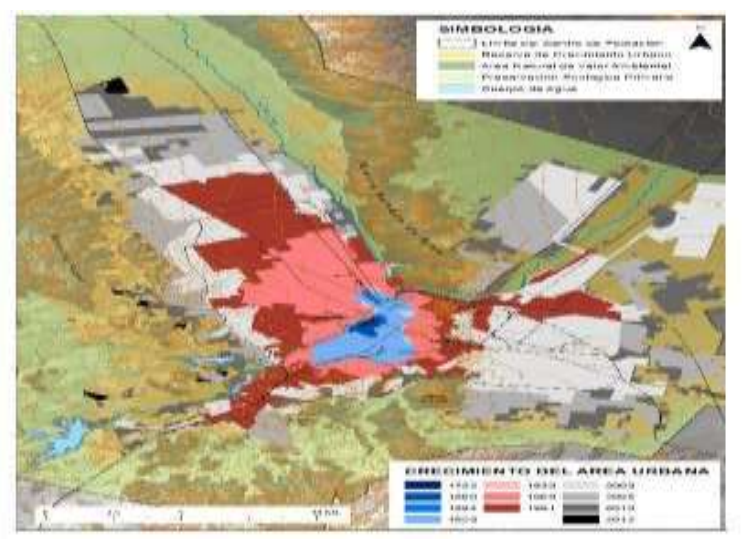

Figura 3 Evolución del Crecimiento urbano, Fuente IMPLAN 2012 
Nuevos desarrollos urbanos de la Ciudad de Chihuahua

Se localizaron cuatro de los nuevos fraccionamientos que se han autorizado en los últimos 10 años con mayor problemática en la ciudad, algunos de ellos se ubican en colindancia con Aldama al Noroeste de la ciudad, al sur- oriente, al sur, al poniente, al noroeste y al norte de la ciudad de Chihuahua (ver mapa 2).

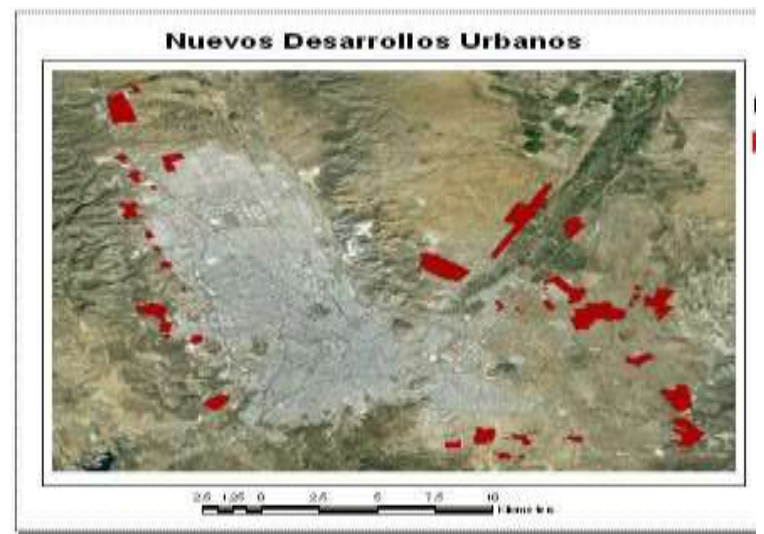

Mapa 2 Nuevos Desarrollos Urbanos de la ciudad de Chihuahua

En los fraccionamientos de nueva creación del muestreo que se realizó, se analizaron las condiciones en las que se encuentra cada asentamiento desde su infraestructura, accesibilidad a los individuos, servicios establecidos, equipamiento, entre otras cuestiones (ver anexos tabla 1).

\section{Fraccionamiento Haciendas del Rejón I}

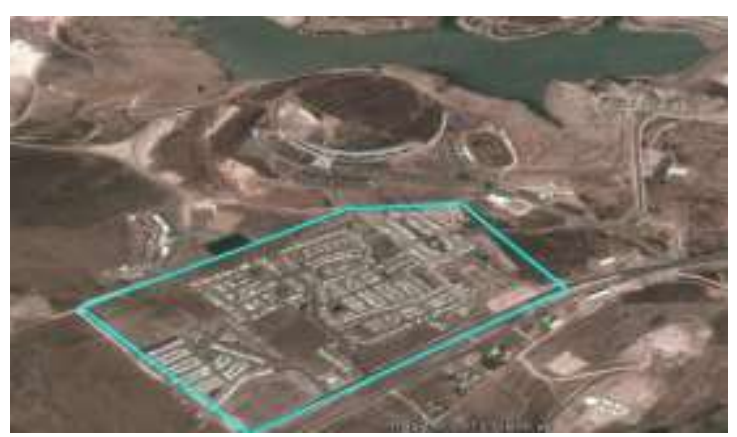

Imagen 1
Fraccionamiento Riveras del Sacramento

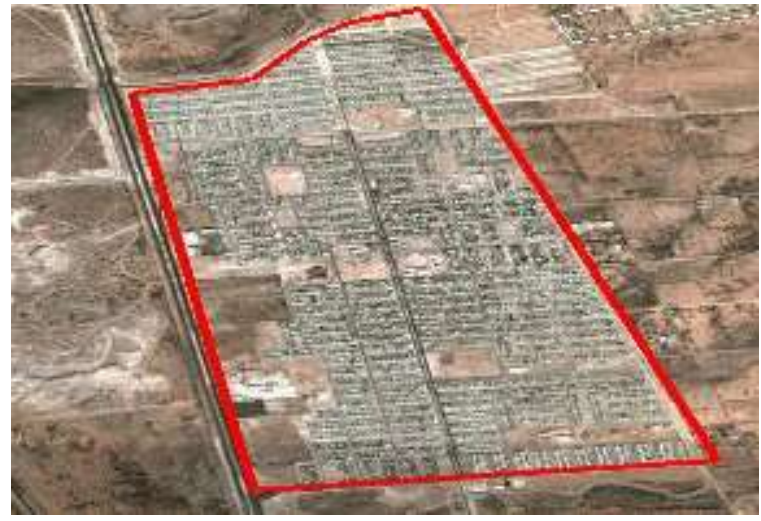

Imagen 2

\section{Fraccionamiento Paseos de Camino Real}

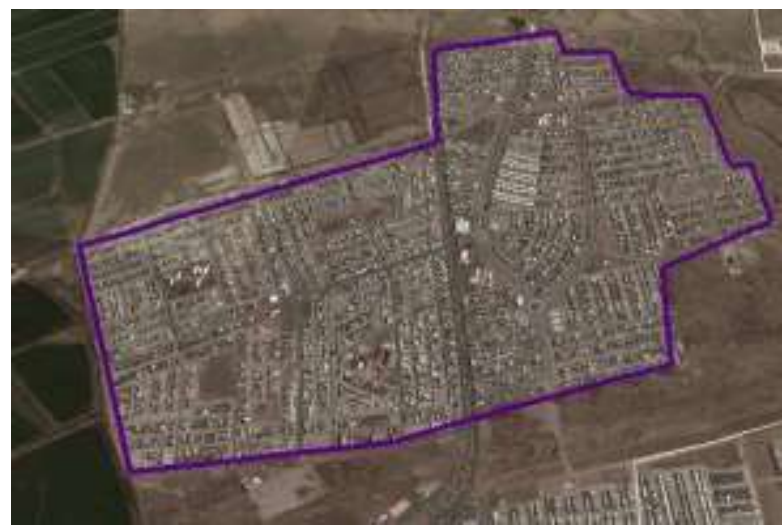

Imagen 3

\section{Fraccionamiento La Cantera}

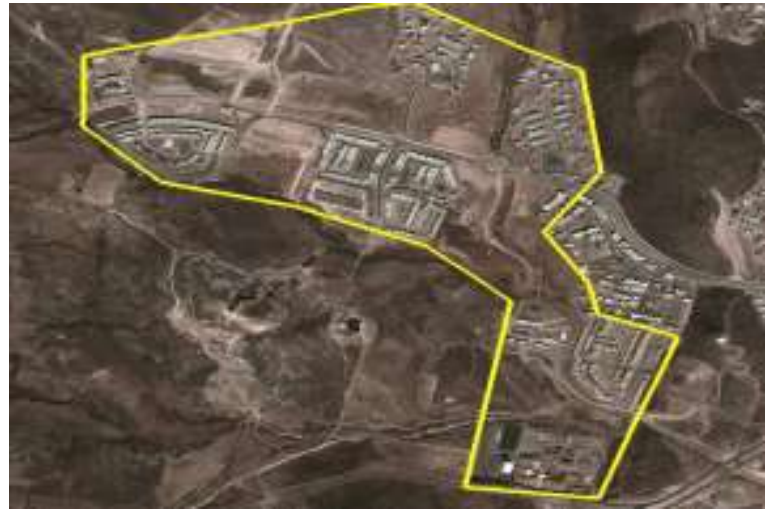

Imagen 4 


\section{CONCLUSIONES}

La Ordenación Territorial de una Ciudad es muy compleja, pero podemos ir realizando cambios de acorde a un plan que se adecue a las situaciones y recursos de la Ciudad y la población.

Con el análisis del muestreo de los fraccionamientos se observó que el auge de los fraccionamientos cerrados limita el acceso tanto interno como externo al área, son creados sin el equipamiento y los servicios necesarios, se asientan en usos de suelo incompatible para residir y dichos fraccionamientos se aprueban sin tener las características idóneas para una buena calidad de vida.

El énfasis que se le ha dado a la zona norte con accesos viales, equipamiento; como escuelas, hospitales, centros comerciales, dándole plusvalía a las terrenos comerciales, habitacionales e industriales, tomando como una mejor opción el vivir hacia la zona norte de la ciudad dejando olvidada a la zona sur, marcando una gran desigualdad entre los habitantes y los servicios que se encuentran de un lugar al otro, teniendo como consecuencia un crecimiento desordenado, dejando espacios vacíos, problemas de inseguridad, fraccionamientos no habitados generando delincuencia, largas distancias, estrés entre la gente, ciudades dormitorio, usos de suelos inadecuados, creación de nuevas fuentes de empleo, entre otros.

Lo que todo esto ha llevado a que el gobierno le cueste más llevar todos los servicios a dichos fraccionamientos, teniendo mayores impuestos para la población, desapropiación del espacio en el que se vive, entre otros que forman una larga lista de problemas, y todo por no tener una planeación adecuada del territorio que el gobierno gestiona o normaliza.

\section{LITERATURA CITADA}

II Conteo de Población y Vivienda INEGI. 2005. Consultado en Agosto 2013. http://www.inegi.org.mx/prod_serv/contenid os/espanol/bvinegi/productos/integracion/mu nicipios/mexympios/MexIICona_6.pdf

Fernández Giiell José Miguel. Planeación Estratégica de Ciudades. Barcelona. 2006. Editorial Reverte. Estudios Universitarios de Arquitectura. Pág 148.

Gómez Orea Domingo. Ordenación Territorial. Madrid. 2007. Editorial Aedo. Pág 32 - 36, 43- 45.

Instituto Municipal de Planeación. Ayuntamiento de Chihuahua. 2010- 2013. Consultado Abril 2013. http://www.implanchihuahua.gob.mx/

Instituto Municipal de Planeación. Plan de Desarrollo Urbano 2040. Consultado en

Septiembre 2013. http://www.implanchihuahua.gob.mx/PDU20 40/psmus/DIAGNOSTICO_URBANO.pdf

Instituto Nacional de Estadística y Geografía (INEGI) Consultado en Agosto 2013 http://cuentame.inegi.org.mx/poblacion/defau lt.aspx?tema $=\mathrm{P}$

Lacomba Ruth. La Ciudad Sustentable, Creación y Rehabilitación de Ciudades Sustentables. México. 2004. Editorial Trillas. Primera Edición. Enero 2004. Pág 140 http://www.diputados.gob.mx/LeyesBiblio/p df/133.pdf

Peña Juan. Cartografía de las Coberturas y Usos de Suelo de la Marina Baixa (Alicante). 2005. Investigación Geográfica

(Esp) Num 33. Revista Redalyc. 
http://www.redalyc.org/articulo.oa?id=17612 746004

San Miguel Villegas Ruth Trinidad. La Expansión Urbana en Suelo de Conservación en la Delegación Tlahuac, D.F México. 2010 Flacso México. Tesis
Italia. México. 2008. Universidad Autónoma de México.

Zorrilla Arena Santiago. Como Aprender Economía, Conceptos Básicos. México. 2004. Editorial Limusa S.A.DE C.V. Pág 25.

Torres Felipe. Formaciones Regionales

Comparadas: Los casos de México, España e

\section{ANEXOS}

Características de los fraccionamientos analizados

\begin{tabular}{|c|c|c|c|c|}
\hline $\begin{array}{c}\text { Fracciona } \\
\text {-miento }\end{array}$ & Vivienda & Servicios & Infraestructura Vial & Equipamiento \\
\hline $\begin{array}{l}\text { Hacienda } \\
\text { s del } \\
\text { Rejón }\end{array}$ & $\begin{array}{l}\text { Creado en 2010, ubicado a } \\
\text { espaldas de la Presa Rejón, } \\
\text { vivienda de altos ingresos, } \\
\text { residencial } \\
\text { construcción amplia. }\end{array}$ & \begin{tabular}{|l|} 
No existen \\
rutas de \\
transporte \\
público, \\
servicios \\
básicos
\end{tabular} & $\begin{array}{l}\text { Cambio de uso de suelo de } \\
\text { área natural a habitacional } \\
\text { y de servicios, se cuenta } \\
\text { solo con un acceso al } \\
\text { fraccionamiento que es la } \\
\text { Ampliación de la Av, } \\
\text { Teófilo Borunda. }\end{array}$ & $\begin{array}{l}\text { Se han ido creando } \\
\text { algunas instituciones } \\
\text { educativas y algunos } \\
\text { comercios, teniendo } \\
\text { un gran auge por la } \\
\text { cercanía a la Presa El } \\
\text { Rejón. }\end{array}$ \\
\hline $\begin{array}{l}\text { Riveras } \\
\text { de } \\
\text { Sacramen } \\
\text { to }\end{array}$ & $\begin{array}{l}\text { Ubicado en la Carretera } \\
\text { Chihuahua-Sacramento, } \\
\text { creado en 2006, anteriormente } \\
\text { eran ejidos, creando vivienda } \\
\text { de interés social con muy poca } \\
\text { construcción. }\end{array}$ & $\begin{array}{l}\text { Servicios } \\
\text { básicos, } \\
\text { transporte } \\
\text { público pero } \\
\text { deficiente. }\end{array}$ & $\begin{array}{l}\text { Cambio de uso de suelo } \\
\text { ejidal a habitacional, con } \\
\text { un solo acceso que es la } \\
\text { carretera } \\
\text { Sacramento. Chihuahua- }\end{array}$ & \begin{tabular}{|lr|}
\multicolumn{3}{|l|}{ Solo existen algunas } \\
escuelas de & nivel \\
básico, de & otros \\
equipamientos & no \\
existen. &
\end{tabular} \\
\hline $\begin{array}{l}\text { Paseos del } \\
\text { Camino } \\
\text { Real }\end{array}$ & $\begin{array}{l}\text { Ubicado a un costado del } \\
\text { aeropuerto internacional de } \\
\text { Chihuahua, creado en 2005, } \\
\text { usos ejidales, un existen } \\
\text { parcelas de ejidos, viviendas } \\
\text { de interés social con muy } \\
\text { pocos metros de construcción. }\end{array}$ & $\begin{array}{l}\text { Servicios } \\
\text { básicos, } \\
\text { transporte } \\
\text { público pero } \\
\text { deficiente. }\end{array}$ & $\begin{array}{l}\text { Solo se cuenta con un } \\
\text { acceso que es el Blvd. Juan } \\
\text { Pablo II y la calle principal } \\
\text { Av. Equss. }\end{array}$ & $\begin{array}{l}\text { Antes de la creación } \\
\text { del fraccionamiento } \\
\text { ya se contaba con } \\
\text { algunas industrias } \\
\text { maquiladoras, existía } \\
\text { ya una planta } \\
\text { tratadora de agua, } \\
\text { algunas escuelas de } \\
\text { nivel básico. }\end{array}$ \\
\hline $\begin{array}{l}\text { La } \\
\text { Cantera }\end{array}$ & $\begin{array}{l}\text { Creado en 2008, ubicado en el } \\
\text { noreste de la ciudad, } \\
\text { viviendas de amplia } \\
\text { construcción con nivel medio- } \\
\text { alto, cerrado y privado. }\end{array}$ & $\begin{array}{l}\text { Cuenta con } \\
\text { los servicios } \\
\text { básicos, pasa } \\
\text { el transporte } \\
\text { público } \\
\text { cerca del } \\
\text { fraccionamie } \\
\text { nto. }\end{array}$ & $\begin{array}{l}\text { Se cuenta con un principal } \\
\text { acceso que es la Av. De la } \\
\text { Cantera, con futuros } \\
\text { accesos. }\end{array}$ & $\begin{array}{l}\text { Se encuentra en una } \\
\text { buena ubicación de la } \\
\text { ciudad ya que tiene } \\
\text { todos los servicios y } \\
\text { equipamientos } \\
\text { cercas. }\end{array}$ \\
\hline
\end{tabular}

Tabla 1 Características de los Fraccionamientos Analizados. 
Copyright (c) 2017 Clara Patricia Orozco González, Damián A. Porras Flores, Anabel Ortega Rodriguez

Ricardo A González Aldana y Cesar G G arcia G onzález

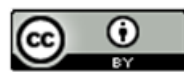

Este tex to está protegido por una licencia licencia Creative Commons 4.0.

Usted es libre para Compartir —copiar y redistribuir el material en cualquier medio o form ato- y Adaptar el documento —remezclar, transformar y crear a partir del material一 para cualquier propósito, , incluso para fines comerciales, siempre que cumpla la condición de:

Atribución: Usted debe dar crédito a la obra original de manera adecuada, proporcionar un enlace a la licencia, e in dicar si se han realizado cam bios. Puede hacerlo en cualquier form a razonable, pero no de form a tal que sugiera que tiene el apoyo del licenciante o 10 recibe por el uso que hace de la obra.

Resumendelicencia - Textocompletodelalicencia 